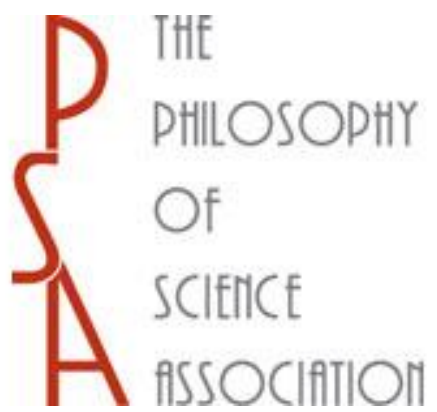

\title{
Probabilistic Causality and Multiple Causation
}

Author(s): Paul Humphreys

Source: PSA: Proceedings of the Biennial Meeting of the Philosophy of Science Association, Vol. 1980, Volume Two: Symposia and Invited Papers (1980), pp. 25-37

Published by: The University of Chicago Press on behalf of the Philosophy of Science Association

Stable URL: https://www.jstor.org/stable/192584

Accessed: 23-05-2020 00:47 UTC

JSTOR is a not-for-profit service that helps scholars, researchers, and students discover, use, and build upon a wide range of content in a trusted digital archive. We use information technology and tools to increase productivity and facilitate new forms of scholarship. For more information about JSTOR, please contact support@jstor.org.

Your use of the JSTOR archive indicates your acceptance of the Terms \& Conditions of Use, available at https://about.jstor.org/terms

The University of Chicago Press, Philosophy of Science Association are collaborating with JSTOR to digitize, preserve and extend access to PSA: Proceedings of the Biennial Meeting of the Philosophy of Science Association 


\title{
$\underline{\text { Probabilistic Causality and Multiple Causation }}{ }^{1}$
}

\author{
Paul Humphreys
}

The University of Virginia

I shall argue in this paper that although much attention has been paid to causal chains and common causes within the literature on probabilistic causality, a primary virtue of that approach is its ability to deal with cases of multiple causation. In doing so I shall try to indicate some ways in which contemporary sine qua non analyses of causation are too narrow (and ways in which probabilist ic causality is not) and refine an argument by Reichenbach designed to provide a basis for the asymmetry of causation.

1. The Scope of Probabilistic Causation

In his contribution to this symposium (Salmon 1981) Professor Salmon has emphasized the central role played by processes and interactions in causality. Whereas his discussion of common causes naturally has a futuristic orientation using causal fans of the type given in Fig. 1.,

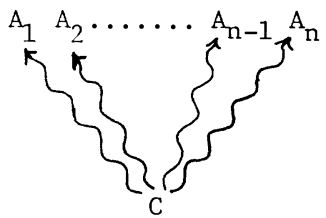

Fig. 1

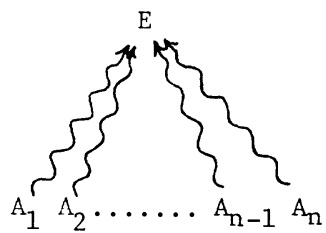

Fig. 2

I shall be rather more backwards-looking and concentrate on the advantages that probabilistic causation possesses for the analysis of multiple causes of the type ${ }^{2}$ in Fig. 2 .

Appropriately, history supports such an orientation. In the early

PSA 1980, Volume 2, pp. 25-37

Copyright (C) 1981 by the Philosophy of Science Association 
years of the twentieth century statistics was, to a considerable body of practitioners, the study of multiple causation. George Udny Yule in his classic textbook of $1911^{3}$ provided this illuminating definition: "The theory of statistics is the exposition of methods specially adapted to the elucidation of quantitative data affected by a multiplicity of causes." (Yule 1911, p.5). Supporting this claim was a careful exposition of measures of association which, stripped of its archaic notation, is closely related to the early part of Suppes' 1970 monograph (Suppes 1970). That causal tradition in statistics later became overshadowed by the theory of statistical inference, but it has been picked up by those earliest users of statistics, the sociologists. In the course of this evolution, the methods shifted from probabilistic theories of causality to theories of probabilistic causality, as statistical methods of data analysis gave way to probabilistic models incorporating the causal processes which connect the relevant variables. This reorientation, which has been noticed in the philosophical 1iterature by Deborah Rosen (Rosen 1980) is typified by this quotation from Otis Dudley Duncan's book on structural equation techniques: "Here we distinguish sharply between (1) statistical description, involving summary measures of the joint distribution of observed variables... and (2) statistical methods applied to the problem of estimating coefficients in a structural model...one cannot even get started on the latter task without a firm grasp of the relevant scientific theory, because the starting point is, precisely, the model and not the statistical methods." (Duncan 1975, pp. 5-6). Noting first that Duncan is following standard sociological practice in using "model" to refer to an (interpreted) theory, the emphasis on theories illustrated by the quotation is, I believe, vitally important because of the fact that the use of probabilistic causality (as distinct from the statistical analyses mentioned earlier) seems to commit us to indeterminism. We must not lose sight of the fact that scientific causal claims are, for the most part, made not about the phenomena theinselves, but models of it (where "model" is now used in the standard philosophical way or, as I would prefer, in some more robust Campbellian sense, in which structural relations and processes are certainly not obviously settheoretic entities). Most philosophical theories of causation, in the course of being justified by appeal to commonplace examples, tacitly rely on this too, for it surfaces in their standard assumption of determinism. The concepts of determinism and indeterminism can be made rigorous only by recourse to deterministic theories and models, and indeed determinism is plausible only if we do, even implicitly, refer our claims to these models, for the vast weight of everyday experience is against determinism. (Indeterminism, of course, is an extremely weak hypothesis, it needing but a single process to go awry for indeterminism to hold.) Yet as soon as we revert to models representing even moderately sophisticated phenomena, they turn out to be indeterministic. I take as my reference point here the wide use of causal theories in the sociological, economic, and biological literature where, despite a methodological commitment to determinism, the models themselves are actually indeterministic without their ad hoc "error terms". 4 It is not just that an enormous gain in simplicity is thereby achieved by moving to these abstract models (for example, in 
the five variable case alone, there are over one thousand possible causal models) but that as Duncan stresses in the above quotation, in non-experimental contexts we should never know where to begin without the model. The point is that were we to rule out such claims made on the basis of indeterministic models as genuinely causal, we should have to reject almost all causal claims that we make. The common use of such models also provides a reason for abandoning the emphasis on causal chains which is so prevalent in the philosophical literature on causation. Chains are simply a limiting case of the general situation of multiple causation. Thus, whereas Salmon wishes to reject causal chains because of their reliance on a basic event ontology (Salmon 1981, Section 8), I should place at least as much emphasis on the inability of the chain approach to adequately represent situations involving multiple causal influences. As Cartwright (1979) has observed, the consequences of such multiple influences are not always simply the combined results of the individual effects, and the use of non-linear "interactive terms" included in structural equations, for example, recognizes that.

No claim is being made here for such causal models being ontologically fundamental of course, and I have specifically avoided references to models of quantum mechanical phenomena. The additional difficulties imposed by the indistinguishability of many elementary particles and the problems associated with locality require separate analysis. It is also important, I believe, to make it clear when we are doing metaphysics and when philosophy of science. The present section of this paper falls squarely into the latter category.

We can do more than merely appeal to causal usage in science, however, when comparing probabilistic causality with alternative accounts. Probabilistic causality can be counted as a species of causation by virtue of satisfying a basic principle of causality - the principle of relevant difference. This principle asserts that for something to be a cause of an effect, it must make some difference to that effect. By insisting on a strict criterion of event identity, this can be put as: the presence of a cause must make some difference, within the circumstances, to whether the effect occurs or not, as compared to the cause's absence. This is obviously not a sufficient condition for something to be a cause (it allows self-causation and omits a criterion of causal direction) but it 1 ies at the heart of many causal analyses. In particular, it is recognized by sine qua non accounts: "The intuitive idea behind [the counterfactual analysis] is fairly simple. To say that the event $c$ is the cause of the event $e$ is to say that the occurrence of event e depended in some way on the occurrence of event c." (Swain 1978 , p. 1) (The author is actually discussing a theory of causation which insists that a cause be necessary for the effect. Counterfactual accounts of causation can be wider than that, though sine qua non and counterfactual approaches are usually identified. I shall avo id that identification and use the sine qua non terminology.) Application of the principle of relevant difference supports our doubts about overdetermining causes as cases of proper causation, and along with these doubts, the rejection of the simple sufficiency view of causation. The 
manipulability account also appears to satisfy the principle.

Because causal theories which insist that a cause must be necessary in the circumstances for the effect have enjoyed wide use, and moreover seem to have the ability to include indeterministic causal sequences, I shall devote the remainder of this section to illustrating their inability to provide correct answers (or any answers at all) in many cases of multiple causation. Necessary conditions are simply a special case of probabilistic causes, for if $A$ is necessary for $E$, then $A$ raises the probability of $E$, and hence is a contributing cause when there is a process connecting the two. Not all contributing causes are necessary, as we shall see, and sine qua non accounts are also incomplete in failing to include counteracting causes (in the sense used here). In what follows, I shall restrict the discussion to situations involving contributing causes.

The two central features of the theory of probabilistic causality adopted here are that (1) a cause changes the probability of the effect and (2) a completed process connects cause and effect. 5 It is clear that (1) satisfies the principle of relevant difference - contributing causes by making the effect more probable and counteracting causes by making it less probable. Equally important is the fact that probabilistic causes can satisfy this principle even in cases of multiple causation. Consider the situations diagrammed below, where a set of background conditions $F$ is assumed to hold in all three cases. Fig. 3a is here to represent the situation where A individually, or B individually, is capable of producing $E$, even though they are not sufficient for E. Because when both $A$ and $B$ occur, with completed processes running between $A$ and $E$, and between $B$ and $E$, $A$ is prima facie not necessary in the circumstances for $E$, and neither is $B$, and so such a situation is as potentially difficult to incorporate into a sine qua non analysis as is the much discussed overdetermination case diagrammed in Fig. 3b.

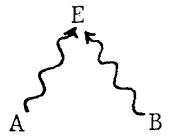

Fig. 3a

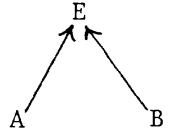

Fig. 3b

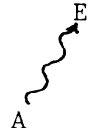

$\mathrm{B}$

Fig. $3 c$.

Cases of the kind sketched in Fig. 3a frequently occur. For example, we may apply both heat and incident 1 ight to a metal so that the phenomena of thermionic and photoelectric emission are both operating. Neither the heat nor the light source is necessary in the circumstances for the common effect of an electron emission when it occurs (nor indeed is either sufficient). From the social sciences, a second example occurs when an individual's economic level and degree of formal 
education supplement one another to cause a favorable attitude towards them from a second individual. A third example familiar from everyday experience is that of a car whose weak spark and faulty carburetion could each be responsible for the car's failure to start. Again, neither is necessary for the common effect when both are present. In such cases, as long as the condition $\mathrm{P}(\mathrm{E} / \mathrm{ABF})>\mathrm{P}(\mathrm{E} / \overline{\mathrm{A}} \mathrm{B} F)$ is satisfied, A will count as a contributing cause of $E$, even given the presence of $B$, and the same holds, mutatis mutandis, for B. Although I am firmly convinced, both on the grounds of common usage and satisfaction of conditions (1) and (2) that it is correct to call A a cause of B in such a situation as occurs in Fig. 3a, I shall not use this type of example to argue the merits of probabilistic causality, for most sine qua non analyses do not cover this type of case. By either explicitly (e.g., Lewis 1973 , p. 559) restricting themselves to deterministic situations, or, more commonly, by simply discussing deterministic cases exclusively, cases of the kind represented by Fig. 3a have not been raised in the 1 iterature, the focus rather being on overdetermination situations of the type given in Fig. 3b. (One notable exception here is Mackie (1974), whose theory I discuss below.)

Before leaving this example, I must emphasize that the superficial similarity to overdetermination cases should not mislead us into thinking that the present case suffers from the difficulties of that much discussed 6 situation of $\mathrm{Fig} .3 \mathrm{~b}$. The fact that a completed process connects cause with effect does not mean that invariably the probability of the effect steadily increases until, right before it occurs, it has the value one. This does indeed hold for some processes, but to believe it true in general is to fall prey to a "chicken counter's fallacy" in which an incorrect image of the causal process heading towards the effect's fixed point of occurrence plays a large role. The (conditional) probability of the effect can easily decrease as can be seen by considering a process in which $\overline{\mathrm{E}}$ becomes progressively more probable, yet E eventually occurs. The (conditional) probability of the effect can also stay constant across time. Thus an indeterministic effect can be uncertain right up to the point at which it actually occurs, and so is not overdetermined, even when multiple causal influences are present. I should also point out that whereas genuine cases of overdetermination are rare (overdetermining causes usually alter the effect while supplementary causes, for example, can simply increase the chance of the effect without materially altering it) the examples that I 1 isted above for the indeterministic case are not. Many other examples could be cited.

To more sharply see the difference between an account which insists on a cause being necessary for the effect and the present approach, consider now a situation 1 ike that of $\mathrm{Fig}$. 3c. Here A, B occur simultaneously, $P(E / A B F)>P(E / \overline{A B F})$ but now no completed process connects $B$ with $E$. $B$ is insufficient in the circumstances for $E$, although it could by itself produce $\mathrm{E}$ on occasion. I shall consider the case where $\mathrm{A}$ is also insufficient, although the argument holds when $P(E / A F)=1$. Finally, $E$ is not allowed to occur spontaneously. Now, of course the specific answer given by a sine qua non analysis for this case will depend upon 
the particular semantics for counterfactuals used. Because Mackie's account has explicitly included indeterministic situations under its scope, I shall, for concreteness, discuss that theory here. I believe, however, that this type of case provides serious difficulties for all existing sine qua non theories.

Whereas there might be residual doubts that $A$ is actually a cause of $E$ in situation $3 a$, there surely $c$ an be no question that $A$ is a cause (perhaps even the cause) of $E$ in situation $3 c$ and that $B$ was not. Yet Mackie's analysis leads to the claim that $A$ was not a cause of $E$ in circumstances 3c. His counterfactual analysis (1974, Ch. 2, especially pp. 33, 52; although see also the caveat about processes on p. 86) requires that for $A$ to be a cause of $E$ the counterfactual "If $A$ had not occurred, then in the circumstances, E would not have occurred" must be true. We test for the truth of that sentence by considering a situation which is exactly similar up to the point at which A occurs, then removing $A$ and replacing it with some "neutral" event. Then we let the world run on according to the prevailing laws. If we can then assert that $E$ would not occur in that counterfactual situation, $A$ was a cause of $E$ in the original situation. In the case at hand, removing $A$ will not result in the removal of $B$, because $B$ occurs simultaneously with A, and the world remains the same up to the point at which A occurs. Furthermore B, although not sufficient for E, might, when we let the world run on without $A$, lead to $E$ in the counterfactual situation. The presence of a completed process between $B$ and $E$ in the substitute situation and the absence of a process between the neutral substituting event and $E$ are allowed by the fact that the world runs on in an indeterministic fashion. Hence we certainly cannot assert that in the counterfactual situation, E would not occur. So, under Mackie's theory, $A$ was not a cause of $E$. But if not, what was? In contrast, probabilistic causality allows that because A raised the probability of $E$, and was connected to it by a completed process, $A$ was a cause of $E$, and that, I think, is correct.

There is one special case of "multiple causation" which deserves attention because it affects even isolated causal chains, and that is the case I excluded in the above example, where an event can occur spontaneously. Spontaneity is a particularly puzzling form of aleatory activity because there appears to be nothing which we can ultimately identify as a cause of the spontaneously produced event other than a non-zero chance of its occurrence. Actual examples of this type of situation, where an event can occur either spontaneously or after being induced by some physical stimulus, are a liquid being placed in a freezer, where it also has an infinitesimally small chance of freezing spontaneously; and a laser beam, where there is a practically negligible probability of coherent light being emitted spontaneously rather than by stimulated emission. We can, of course, provide detailed descriptions of the physical structures underlying such phenomena, but that simply pushes the spontaneity to a deeper level. Such spontaneous occurrences can be ruled out as caused under probabilistic causality. The reason is simply that there is no contrast situation available with which to compare the effect of the ever- 
present spontaneity. Even modern treatments of 'spontaneous' occurrences which tend to attribute what was formerly spontaneous to an ineradicable residual field (as for example the treatment of spontaneous emission of radiation in Sakurai (1973) does) appear to suffer from the same lack of a contrast case in that it is physically impossible to eliminate the zero-point field. When the inducing event and process are present as well as the spontaneity, probabilistic causation will allow the inducing event as a cause, as it does with the ordinary multiple causation cases discussed above. But again, sine qua non theories will be hard pressed to allow the inducing event as a cause. The examples just given adequately illustrate, I think, the incorrectness of denying that such events are causes.

\section{The Asymmetry of Multiple Causation}

In section 19 of The Direction of Time (Reichenbach 1956), Reichenbach claimed that the properties of certain types of causal forks allowed a (local) direction of macroscopic causation to be determined. As it stands, the argument is unsatisfying, because it relies on our having previously decided on the basis of microscopic considerations that explanations by final causes are not possible. Nevertheless, the basic idea that we may arrive at a causal asymmetry through multiple causes and effects rather than single sequences is striking. I shall provide here a more detailed argument for causal asymmetry which avoids the appeal to explanations.

Reichenbach's argument can be easily presented. Common causes often satisfy the conditions for a conjunctive fork:

$$
\begin{aligned}
& \text { (i) } \mathrm{P}(\mathrm{AB} / \mathrm{C})=\mathrm{P}(\mathrm{A} / \mathrm{C}) \cdot \mathrm{P}(\mathrm{B} / \mathrm{C}) \\
& \text { (ii) } \mathrm{P}(\mathrm{AB} / \overline{\mathrm{C}})=\mathrm{P}(\mathrm{A} / \overline{\mathrm{C}}) \cdot \mathrm{P}(\mathrm{B} / \overline{\mathrm{C}}) \\
& \text { (iii) } \mathrm{P}(\mathrm{A} / \mathrm{C})>\mathrm{P}(\mathrm{A} / \overline{\mathrm{C}}) \\
& \text { (iv) } \mathrm{P}(\mathrm{B} / \mathrm{C})>\mathrm{P}(\mathrm{B} / \overline{\mathrm{C}})^{7} .
\end{aligned}
$$

Reichenbach claimed that (i) through (iv) are not logically sufficient conditions for a fork to be a common cause for, he asserted (1956, pp. 161-2) it was logically possible for common effects to satisfy the statistical relations as well. Yet, he went on to argue, as a matter of fact, common effects only satisfy the definition of a conjunctive fork when preceded by a common cause. The reason for this is that conditions ( $i$ ) through (iv) jointly entail that $P(A / B)>P(A / \bar{B})$. This statistical association needs to be explained, and only a common cause can do that. Common effects cannot explain the association between their causes, for that would constitute an explanation by a final cause. Hence, he concluded, whenever we came across a fork which is open on one side as in Fig. $3 \mathrm{a}$, and which satisfied the definition of a conjunctive fork, we could infer that it was an instance of a common cause, and hence the direction of causation was away from the node. There are two grounds on which this argument could be faulted. As mentioned above, 
we need an auxiliary argument to substantiate the claim that effects cannot explain their causes, and one which does not presuppose a direction of causation. Equally troubling is the lack of an independent specification of what is to count as a common effect. Two cases are important here. In the first case, referring to Fig. 3a again, as in the discussion of Section 1 both A individually, and B individually, can lead to $E$. Alternatively, it could be the case that neither A nor $B$ is individually capable of producing $E$, but together they can. In this second case, we should allow that A was probabilistically necessary for $E$ in the circumstances, and so was $B$. Reichenbach was never explicit about which of these cases he intended by "common effects", but his examples all appear to be of the second kind. (See, for examples, Reichenbach 1956, p. 157). This is not surprising, for cases in which both $A$ and $B$ are necessary for E trivially satisfy clauses (i), (iii), and (iv) of the definition of a conjunctive fork because when both $A$ and $B$ are necessary ancestors of $E, P(A B / E)=1=$ $P(A / E)=P(B / E)^{8}$. Hence if we are to find cases of common effects which are not conjunctive forks, we must either focus on clause (ii) or look at cases where neither cause is necessary for the effect. I shall do the 1atter. Because such cases are commonly encountered (see the examples given in Section 1 above) our causal analysis of the world would be seriously incomplete were we to omit consideration of them. So with this type of common effect in mind, let us consider the following situation:

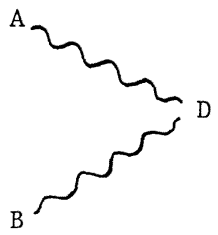

Fig. 4

Here we have a fork $A D B$ whose causal direction is unknown. For it to be a conjunctive fork, the condition

$$
\text { (i) } P(A B / D)=P(A / D) \cdot P(B / D)
$$

has to be satisfied. Now suppose that in the situation at hand,

$$
\text { (II) } \mathrm{P}(\overline{\mathrm{A}} \cdot \overline{\mathrm{B}} / \mathrm{D})=0 \text {. }
$$

If the situation is a common effect, so that A and B causally precede D, condition (II) says that A, B are the only events capable of producing D. In many situations, this condition will be satisified.

Also suppose that

(III) $\mathrm{P}(\mathrm{A} / \mathrm{D})<1$ and $\mathrm{P}(\mathrm{B} / \mathrm{D})<1$. 
Again, if the situation is one of a common effect, this means that neither $A$ nor $B$ is individually necessary for $D$. Hence when both (II) and (III) hold and the direction of causation is from $A$ to $D$ and from $B$ to $D$, we have a common effect whose antecedents exhaust all possible causes of the effect, yet whose joint presence precludes either from being individually necessary for that effect. Can condition(i) be satisfied by such a common effect? Suppose it were. Then by the algebraic properties of probabilistic independence, (i) and (II) give

$$
0=P(\bar{A} \cdot \bar{B} / D)=P(\bar{A} / D) \cdot P(\bar{B} / D),
$$

and hence either

$$
P(\bar{A} / D)=0 \text { or } P(\bar{B} / D)=0 \text {, }
$$

violating (III). So when (II) and (III) are satisfied by a common effect, that effect cannot be a conjunctive fork.

of course, (i), (II) and (III) are inconsistent in the case of common causes too. But whereas there are cases of common effects where (II) and (III) are satisfied, and hence (i) violated, when we have common causes it is (II) that will often be false in indeterministic situations. When D causally precedes $A$ and $B$, (III) simply says that $D$ is insufficient for $A$ and for $B$. Thus $D$ can be followed by both $\bar{A}$ and $\bar{B}$, making (II) false unless there is some interaction between $\bar{A}$ and $\bar{B}$ precluding their joint occurrence. If not, then (II) will be false and (i) can be satisfied.

These considerations show that when a fork satisfies (II) and (III) above, it is either a common effect or an interactive common cause. To decide which is the case, we must leave logic and turn to the kinds of considerations outlined by Salmon in section 7 of his paper (Salmon 1981) - the claim that interactive common causes can be distinguished from other kinds of common causes by the nature of the laws governing them. Although we have here an argument for a local causal asymmetry only, the discussion indicates that, contrary to Reichenbach's claim, some types of common effects cannot be conjunctive forks whether preceded by common causes or not. Furthermore, if Salmon's contentions about interactive common causes are correct, the appeal to fundamental physical laws could be made without necessarily retreating to microstatistical considerations. As long as there are fundamental physical laws governing macroscopic phenomena which are not supervenient upon microscopic laws, a purely macroscopic account of the direction of causation could be given.

By way of a final remark, the argument given by van Fraassen in (1977) which claims to show that the demand for common causes rules out indeterministic theories can be reconstructed in terms of our three conditions (i), (II) and (III). He considers a situation where an event $C$ is invariably followed by one of the three incompatible events $A, B, D$. Letting $\varnothing$ be (A or D) and $\psi$ be ( $B$ or $D$ ), he finds that $C$, although a complete specification of the state of the system, does 
not satisfy $P(\emptyset . \psi / C)=P(\emptyset / C) \cdot P(\psi / C)$. One might point to the fact that $\emptyset$ and $\psi$ co-occur only when D occurs as one of the reasons why $\emptyset$ and $\psi$ are not conditionally independent on $C$. A more interesting point is that $\emptyset, \psi$, and $C$ satisfy our condition (II) (with $\emptyset$ replacing $A, \psi$ replacing $B$, and $C$ replacing $D$ ) and hence if we were to insist on condition (i) being satisfied, as Reichenbach's principle of the common cause does (see, Reichenbach 1956, section 19), we should have to reject (III), i.e., require that $P(\emptyset / C)=1$ or $P(\Psi / C)=1$. C would thus have to determine at least one of those events. That of course would not give complete determinism, particularly since $\emptyset$ and $\psi$ are disjunctions of elementary events. The obvious way out of the dilema, however, is simply to note that $C$ is still a common cause of $\emptyset$ and $\psi$, but that retaining (II) and (III) requires accepting $\emptyset \mathrm{C} \psi$ as an inter-. active fork, rather than a conjunctive fork.

\section{Summary}

I have merely been able to scratch the surface of probabilistic causality here. Some questions which I have left untouched are the use of probabilistic causality in decision making; the connection that positive causal relevance has to functional causation and explained variance techniques of statistics; and how the process approach works when something is caused by a process not going to completion - as when my car stops because the electricity shorts out, cutting off current to the distributor. I am optimistic that a11 these have answers which are not too far away from the basic ideas of contributing and counteracting causes. Probabilistic explanation is perhaps the area which stands to benefit most from a correct theory of probabilistic causality, and I believe that the successes already achieved in that area are proof enough of the value of this approach. For a treatment of explanation using the ideas underlying this paper, see Humphreys (1981) and, for a rather different approach, Salmon (1978).

Probabilistic causality thus satisfies three important criteria. The claims that it makes are consistent with causal claims made by wellentrenched scientific theories in ways that existing alternatives are not. It is based upon a principle which, in different ways, motivates other generally accepted causal theories. And it is philosophically fruitful in providing plausible answers to a number of traditional problems associated with causation.

One moral can be drawn from this paper, and that is how the hygienic models of the physical sciences have unduly influenced causal discussion, so that multiple causation has been relegated to a secondary position. This area might, perhaps, be one where the methodology of the social sciences can finally make a distinctive contribution.

\section{Notes}

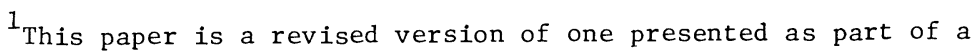


symposium on probabilistic causality. The other symposiasts were Nancy Cartwright and Wesley Salmon. The original paper was read in my absence by James Fetzer.

\section{2}

2 From now on, I shall discuss only the case $n=2$. All arguments can be extended to the general case by obvious methods.

${ }^{3}$ This work, later co-authored with M.G. Kendall, is one of the longest-1ived statistics texts ever, running to the same fourteen editions as Fisher's "Statistical Methods" over a period of seventy years.

${ }^{4}$ A clear statement of the correct use of these models can be found in Blalock (1962), pp 183-184. Further representative remarks on this subject can be found in Simon (1953), pp. 49-52.

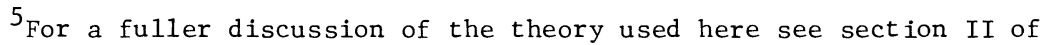
Humphreys (1980).

${ }^{6}$ See, e.g., Lewis (1973), Loeb (1974), Swain (1978), Goosens (1979).

${ }^{7}$ Following Reichenbach, all probabilities in this section are to be interpreted as relative frequencies. By applying these to the single case, this will avoid the difficulty faced by conditional propensities that they already contain a causal direction - and allow us to freely use inverse probabilities.

${ }^{8}$ Most of Reichenbach's examples of common causes are also of this limiting kind. See also Salmon's discussion of perfect forks in Salmon (1981). 


\section{$\underline{\text { References }}$}

Blalock, H.M., Jr. (1962). "Four-Variable Causa1 Models and Partial Correlations." American Journal of Sociology 68: 182-194.

Cartwright, N.D. (1979). "Do the Laws of Physics State the Facts?" Pacific Philosophical Quarterly 61: 75-84.

Duncan, O.D. (1975). Introduction to Structural Equation Models. New York: Academic Press.

Goosens, W.K. (1979). "Causal Chains and Counterfactuals." Journal of Philosophy 76: 489-495.

Humphreys, P.W. (1980). "Cutting the Causal Chain." Pacific Philosophica1 Quarter1y 61: 305-314.

232.

(1981). "Aleatory Explanation." Synthese 48: 225-

Lewis, D.K. (1973). "Causation." Journal of Philosophy 70: 556-567. (As reprinted in Sosa, E. (ed.). (1975) Causation and Conditionals. Oxford: Oxford University Press. Pages 180-191.).

Loeb, L. (1974). "Causal Theories and Causa1 Overdetermination." Journal of Philosophy 71: 525-544.

Mackie, J.L. (1974). The Cement of the Universe. Oxford: Oxford University Press.

Reichenbach, H. (1956). The Direction of Time. Berkeley: University of California Press.

Rosen, D.A. (1980). "A Critique of Deterministic Causality." Unpub1 ished manuscript.

Sakurai, J.J. (1973). Advanced Quantum Mechanics. Reading: AddisonWesley.

Salmon, W.C. (1978). "Why ask 'Why?'?" Proceedings and Addresses of the American Philosophical Association 51: 683-705. (As reprinted, with minor changes, in Salmon, W.C. (ed.). (1979). Hans Reichenbach: Logical Empiricist. Dordrecht: D. Reidel. Pages $\overline{403-}$ 425.)

- "Causality: Production and Propagation." In PSA 1980, Volume 2. Edited by P. Asquith and R. Giere. East Lansing: Philosophy of Science Association. Pages 49-69.

Simon, H. (1953). "Causal Ordering and Identifiability." In Studies in Econometric Method. Edited by W.C. Hood and T.C. Koopmans. New York: John Wiley. Pages 49-74. 
Swain, M. (1978). "A Counterfactual Analysis of Event Causation." Philosophical Studies 34: 1-19.

Suppes, P. (1970). A Probabilistic Theory of Causality. Amsterdam: North-Holland.

van Fraassen, B.C. (1977). "The Pragmatics of Explanation." American Philosophical Quarterly 14: 143-150.

Yule, G.U. (1911). An Introduction to the Theory of Statistics. London: Griffin and Co. 